\title{
Distinct mechanisms for trans-mediated mobilization of cellular RNAs by the LINE-1 reverse transcriptase
}

\author{
José L. Garcia-Perez, ${ }^{1,4}$ Aurélien J. Doucet, ${ }^{2,4}$ Alain Bucheton, ${ }^{2}$ John V. Moran, ${ }^{1,3,5}$ \\ and Nicolas Gilbert ${ }^{2,5}$
}

\author{
${ }^{1}$ Department of Human Genetics, University of Michigan Medical School, Ann Arbor, Michigan 48109-0618, USA; ${ }^{2}$ Institut de \\ Génétique Humaine, CNRS, UPR 1142, 34396 Montpellier cedex 5, France; ${ }^{3}$ Department of Internal Medicine, University of \\ Michigan Medical School, Ann Arbor, Michigan 48109-0618, USA
}

\begin{abstract}
Long Interspersed Element-1 (LINE-1 or L1) sequences comprise $\sim 17 \%$ of human DNA and ongoing L1 retrotransposition continues to impact genome evolution. The L1-encoded proteins also can mobilize other cellular RNAs (e.g., Alu retrotransposons, SVA retrotransposons, and U6 snRNAs), which comprise $\sim 13 \%$ of human DNA. Here, we demonstrate that the trans-mediated mobilization of non-L1 RNAs can occur by either template choice or template-switching mechanisms. Remarkably, these mechanisms are not mutually exclusive, as both processes can operate sequentially on the same RNA template. Finally, we provide evidence that efficient U6 snRNA retrotransposition requires both ORF1p and ORF2p, providing indirect evidence for the action of ORF1p in U6 snRNA retrotransposition. Thus, we propose that the LINE-1-encoded reverse transcriptase can mediate the retrotransposition of non-L1 RNAs by distinct mechanisms.
\end{abstract}

[Supplemental material is available online at www.genome.org.]

The average human genome is estimated to contain $\sim 80-100$ retrotransposition competent L1s (Sassaman et al. 1997; Brouha et al. 2003). These elements are $\sim 6 \mathrm{~kb}$ in length and contain two open reading frames (ORF1 and ORF2) (Scott et al. 1987; Dombroski et al. 1991). ORF1 encodes a 40-kDa RNA-binding protein (p40 or ORF1p) that possesses nucleic acid chaperone activity (Holmes et al. 1992; Hohjoh and Singer 1996; Martin and Bushman 2001; Martin et al. 2005). ORF2 encodes an 150-kDa protein with demonstrated endonuclease (EN) and reverse transcriptase (RT) activities (Mathias et al. 1991; Feng et al. 1996; Ergun et al. 2004). Both proteins are responsible for retrotransposition in cis (Moran et al. 1996; Wei et al. 2001; Kulpa and Moran 2005), which likely occurs via target-site primed reverse transcription (TPRT) (Luan et al. 1993; Feng et al. 1996; Kazazian and Moran 1998; Cost et al. 2002; Kulpa and Moran 2006).

The LINE-encoded proteins can mobilize other cellular RNAs, and a number of pathways have been proposed to account for this phenomenon (Denison et al. 1981; Van Arsdell et al. 1981; Brosius 1991, 1999; Schmitz et al. 2004; Ohshima and Okada 2005; Hulme et al. 2006). For example, in humans, Alu retrotransposition requires the enzymatic activities encoded by ORF2p (Dewannieux et al. 2003), whereas processed pseudogene formation appears to require activities encoded by both ORF1p and ORF2p (Esnault et al. 2000; Wei et al. 2001). Previous studies indicate that the L1-encoded proteins also mobilize SINE-R/ VNTR/ALU (SVA) elements and certain uracil-rich small nuclear RNAs (snRNAs) (Buzdin et al. 2002, 2003; Ostertag et al. 2003; Bennett et al. 2004; Gilbert et al. 2005; Gogvadze et al. 2005; Wang et al. 2005; Weber 2006). Here, we experimentally dem-

\footnotetext{
${ }^{4}$ These authors contributed equally to this work.

${ }^{5}$ Corresponding authors.

E-mail moranj@umich.edu; fax (734) 763-3784.

E-mail Nicolas.Gilbert@igh.cnrs.fr; fax (33) 4-99-61-99-01.

Article published online before print. Article and publication date are at http:// www.genome.org/cgi/doi/10.1101/gr.5870107.
}

onstrate that the L1-encoded reverse transcriptase can retrotranspose cellular RNAs by discrete mechanisms.

\section{Results}

In silico analysis of small noncoding RNA sequences in the human genome

Previous in silico and in vitro data suggest that the L1 reverse transcriptase can "switch" from its own mRNA to U6 snRNA during TPRT, resulting in the formation of chimeric U6/L1 pseudogenes (Buzdin et al. 2002, 2003; Gilbert et al. 2005; Gogvadze et al. 2005). Similarly, in silico analyses have suggested that the L1 retrotransposition machinery also can mobilize other uracilrich small nuclear RNAs, small nucleolar RNAs, and hY RNAs, which are components of the Ro/SS-A autoantigen by a fundamentally different template choice mechanism (Buzdin et al. 2003; Perreault et al. 2005; Weber 2006). To gain greater insight about how these sequences have impacted the genome, we conducted a BLAST search of the human genome working draft sequence (HGWD) using separate small RNA sequences as queries (see Methods). We restricted the analysis to sequences that presented an E-value of less than $5.0 \times 10^{-27}$, as they are $<10 \%$ divergent from the sequence of the functional gene.

One-hundred-ninety-seven U6 snRNA sequences met these criteria. These candidates then were inspected manually to characterize the sequences flanking the paralogous U6 copies. Three sequences were discarded because they either were part of a genomic duplication (two instances) or were contained within an unassigned cosmid (one instance). Approximately 90\% of U6 sequences (173 instances) were flanked at their 3 ' end by a retrotrotransposon. Of these, $78 \%$ (135 instances) had sequence characteristics suggesting that they were interspersed by retrotransposition (i.e., the presence of variable-sized target site duplications [TSDs] and a 3' poly[A] tail). Consistent with previ- 
ous analyses, we identified 74 U6/L1 and 17 U6/processed pseudogene chimeras (Buzdin et al. 2002, 2003). We also identified 76 U6 sequences that terminated in a poly(A) tail that could have been generated by template switching from the poly(A) tail of a cellular RNA to U6 snRNA or by the retrotransposition of an unusual, polyadenylated U6 snRNA (Table 1). Interestingly, we only identified $15 \mathrm{U6/Alu}$ pseudogenes, despite the fact that $\sim 1.5$ million Alu elements are dispersed throughout the genome (see below). We also identified U6/L1 chimeras in the genomes of other placental mammals and the marsupial Monodelphis domestica (opossum; A.J. Doucet and N. Gilbert, unpubl.). Thus, the above data extend previous analyses (Buzdin et al. 2002, 2003), and suggest that the majority (i.e., $>90 \%$ ) of U6 snRNA sequences in the human genome have been dispersed by retrotransposition.

We next extended our analysis to a cohort of other snRNAs that are used in the splicing of conventional GT-AG (i.e., U1, U2, U4, and U5) or minor AT-AC introns (U11, U12, U4atac, and U6atac), as well as U3 snoRNA, which is involved in ribosome biogenesis. Using the criteria employed in the in silico U6 analysis, we identified numerous paralogs of the snRNA sequences (Table 2). For example, some U1, U2, U4, and U4atac sequences ended in a poly(A) tail and are flanked by TSDs, consistent with the idea that they were mobilized to new genomic locations by the L1-encoded reverse transcriptase (Table 2). Akin to the situation observed for U6/L1 chimeric pseudogenes, it is possible that the retrotransposed U1, U2, U4, and U4atac sequences could have been generated by template switching from the L1 mRNA poly(A) to the snRNA. However, the lack of other L1 sequences $3^{\prime}$ of these retrotransposed copies leads us to speculate that unusual polyadenylated snRNAs (Houseley et al. 2006) occasionally can be used as reverse-transcription templates by the L1 retrotransposition machinery.

Interestingly, 7/16 U6atac snRNAs were followed by L1 sequences and exhibited structural similarities to the U6/L1 processed pseudogenes identified above (Table 2). We also detected a single U3/L1 chimera using the above criteria. However, when we lowered the cutoff value $\left(1 \times 10^{-9}\right)$ we identified $11 \mathrm{U3} / \mathrm{L} 1$ chimeras of 72 sequences examined. Similarly, the same cutoff values detected two U5/L1 chimeras of 50 sequences examined. In both cases, the U3 and U5 sequences were $3^{\prime}$ truncated and then were followed by a 5'-truncated L1. Together, these data suggest that U6 snRNA, and to a lesser extent U6atac snRNA, can serve as templates for the L1-encoded reverse transcriptase more frequently than other snRNAs, but that a template-switching event from L1 mRNA to U3 snoRNA or U5 snRNA can occur during the course of genome evolution, albeit rarely.
Table 1. U6 snRNA pseudogenes in the human genome

\begin{tabular}{|c|c|c|c|c|c|c|c|c|}
\hline Chromosome & Chromosome size & Number & L1 & Alu & Pseudogene & $\operatorname{Poly}(A)$ & Alone & Others \\
\hline 1 & 245522847 & 16 & 8 & & 3 & 5 & & \\
\hline 2 & 243018229 & 11 & 3 & & & 8 & & \\
\hline 3 & 199505740 & 16 & 7 & & & 8 & 1 & \\
\hline 4 & 191411218 & 12 & 4 & & & 6 & 2 & \\
\hline 5 & 180857866 & 10 & 4 & & & 5 & 1 & \\
\hline 6 & 170975699 & 6 & 3 & 1 & & 1 & & 1 \\
\hline 7 & 158628139 & 5 & 2 & 1 & & 1 & 1 & \\
\hline 8 & 146274826 & 13 & 7 & & 1 & 4 & 1 & \\
\hline 9 & 138429268 & 6 & 1 & & 2 & 3 & & \\
\hline 10 & 135413628 & 8 & 4 & & 2 & 2 & & \\
\hline 11 & 134452384 & 8 & 3 & & & 4 & & 1 \\
\hline 12 & 132449811 & 10 & 3 & 1 & & 5 & 1 & \\
\hline 13 & 114142980 & 7 & 3 & & 2 & 1 & 1 & \\
\hline 14 & 106368585 & 5 & 2 & & 1 & 1 & 1 & \\
\hline 15 & 100338915 & $10^{*}$ & 2 & & & 4 & 2 & \\
\hline 16 & 88827254 & 7 & 1 & 2 & 1 & 3 & & \\
\hline 17 & 78774742 & 7 & 2 & & & 3 & 1 & 1 \\
\hline 18 & 76117153 & 7 & 3 & & 1 & 3 & & \\
\hline 19 & 63811651 & 7 & 2 & 1 & 1 & 1 & 2 & \\
\hline 20 & 62435964 & 6 & & & 1 & 5 & & \\
\hline 21 & 46944323 & 0 & & & & & & \\
\hline 22 & 49554710 & 3 & & & & 2 & 1 & \\
\hline $\mathrm{x}$ & 154824264 & 16 & 10 & & 2 & 1 & 3 & \\
\hline Y & 57701691 & 0 & & & & & & \\
\hline Total & 3076781887 & $197^{\star *}$ & 74 & 6 & 17 & 76 & 18 & 3 \\
\hline Total with TSD & & & 57 & 4 & 14 & 60 & 7 & 2 \\
\hline Frequency (\%) & & & 38 & 3 & 9 & 39 & 9 & 2 \\
\hline
\end{tabular}

Columns 1 and 2 indicate the human chromosomes and their respective sizes. Column 3 indicates the number of U6 sequences identified on each chromosome using a BLAST search E-value $<5.0 \times 10^{-27}$. Columns 4 through 7 indicate the number of U6/L1, U6/Alu, U6/processed pseudogene, or U6/poly(A) sequences present on each chromosome. Column 8 (Alone) indicates U6 sequences that were not associated with repetitive sequences (18 instances). Column 9 (Others) indicates U6 sequences associated with other repetitive sequences (e.g., DNA transposon or LTR retrotransposon; three instances). The total number of each class of pseudogene, the number that are flanked by target site duplications (TSD), and the percentage of U6 sequences that the pseudogenes represent are indicated at the bottom of the table. The single asterisk $\left(^{*}\right)$ denotes two U6 sequences that were identified in distinct duplicated sequences. Only one representative U6 sequence within the duplicon was analyzed. The double asterisk (**) denotes a U6 sequence found in an unassigned contig that was not used in the analyses. Thus, 194 U6 sequences were analyzed.

\section{An experimental system to detect snRNA/L1 processed pseudogenes}

To experimentally recapitulate $\mathrm{U} 6 / \mathrm{L} 1$ pseudogene formation, we used an established cultured cell retrotransposition assay. We transfected HeLa cells with a retrotransposition-competent L1 that was tagged with a retrotransposition indicator cassette in its $3^{\prime}$ untranslated region (pJM101/L1.3mneoI) (Dombroski et al. 1993; Sassaman et al. 1997). The retrotransposition cassette consists of a backward copy of the neomycin phosphotransferase gene containing its own promoter and polyadenylation signal (Jensen and Heidmann 1991; Freeman et al. 1994; Moran et al. 1996). The пео gene also is interrupted by an intron in the same transcriptional orientation as the L1. This arrangement ensures that G418-resistant foci will arise only if a spliced L1 mRNA undergoes a successful round of retrotransposition (Fig. 1A).

G418-resistant foci harboring L1 retrotransposition events were grouped into pools that ranged in size from 13 to 65 foci and aliquots of genomic DNA from each pool were used as templates in a series of nested PCRs using primers specific for U6 snRNA (U6s1 and U6s2) and the retrotransposition indicator cassette (210NEOas and 173NEOas; Fig. $1 \mathrm{~A})$. The data indicate that approximately one in 15 G418-resistant foci contain a U6/L1 pseudogene (Fig. 1B). Sequence analysis of 43 independent 
Table 2. Small RNA pseudogenes in the human genome

\begin{tabular}{|c|c|c|c|c|c|c|c|}
\hline Name & Number & L1 & Alu & Pseudogene & Poly(A) & Alone & Others \\
\hline \multirow[t]{2}{*}{ U1 } & 53 & 0 & 1 & 0 & 39 & 13 & \\
\hline & Frequency (\%) & 0.0 & 1.9 & 0.0 & 73.6 & 24.5 & \\
\hline \multirow[t]{2}{*}{ U2 } & 38 & 0 & 2 & 0 & 20 & 16 & \\
\hline & Frequency (\%) & 0.0 & 5.3 & 0.0 & 52.6 & 42.1 & \\
\hline \multirow[t]{2}{*}{ U3 } & 12 & 1 & 0 & 0 & 4 & 7 & \\
\hline & Frequency (\%) & 8.3 & 0.0 & 0.0 & 33.3 & 58.3 & \\
\hline \multirow[t]{2}{*}{ U4 } & 23 & 0 & 0 & 0 & 20 & 3 & \\
\hline & Frequency (\%) & 0 & 0 & 0 & 87 & 13 & \\
\hline \multirow[t]{2}{*}{ U5 } & 4 & 0 & 0 & 0 & 0 & 4 & \\
\hline & Frequency (\%) & 0 & 0 & 0 & 0 & 100 & \\
\hline \multirow[t]{2}{*}{ U6 } & 194 & 74 & 6 & 17 & 76 & 18 & 3 \\
\hline & Frequency (\%) & 38.1 & 3.1 & 8.8 & 39.2 & 9.3 & 1.5 \\
\hline \multirow[t]{2}{*}{ U11 } & 2 & 0 & 0 & 0 & 0 & 2 & \\
\hline & Frequency (\%) & 0 & 0 & 0 & 0 & 100 & \\
\hline \multirow[t]{2}{*}{ U12 } & 2 & 0 & 1 & 0 & 0 & 1 & \\
\hline & Frequency (\%) & 0 & 50 & 0 & 0 & 50 & \\
\hline \multirow[t]{2}{*}{ U4atac } & 9 & 0 & 0 & 0 & 5 & 4 & \\
\hline & Frequency (\%) & 0.0 & 0.0 & 0.0 & 55.6 & 44.4 & \\
\hline \multirow[t]{2}{*}{ U6atac } & 16 & 7 & 2 & 0 & 6 & 1 & \\
\hline & Frequency (\%) & 43.8 & 12.5 & 0.0 & 37.5 & 6.3 & \\
\hline
\end{tabular}

Column 1 indicates the name of the small RNA. Column 2 indicates the number of sequences identified in the human genome using a BLAST search E-value $<5.0 \times 10^{-27}$. Columns 3 through 6 indicate the number of Ux/L1, Ux/Alu, Ux/processed pseudogene, or Ux/poly(A) sequences identified in our analyses. Column 7 (Alone) indicates Ux sequences identified in our screen that are not associated with repetitive sequences in their $3^{\prime}$ end. Column 8 (Others) indicates sequences that are associated with other repetitive sequences. The frequency of each category of pseudogene is indicated.

PCR products confirmed the identity of the U6/L1 pseudogenes (Fig. 1C; Supplemental Fig. 1A). The U6 sequence ended in four to eight thymidine residues, which is consistent with the fact that U6 snRNA is transcribed by RNA polymerase III; it was then followed by a variably 5 '-truncated L1 (Fig. 1C; Supplemental Fig. 1A). The data further revealed that there is not a particular consensus sequence/motif in L1 RNA that may facilitate the template switch (Supplemental Fig. 1).

Similar data was observed when the above experiment was conducted with synthetic (L1SM) (Han and Boeke 2004) and natural $\left(\mathrm{TG}_{\mathrm{f}} 21\right)$ (Goodier et al. 2001) mouse retrotranspositioncompetent L1s, consistent with the notion that there is not a particular consensus sequence/motif in L1 RNA that may facilitate the template switch (Supplemental Fig. 2). U6/L1 pseudogenes also could be detected at similar efficiencies (approximately one in 25 G418-resistant foci) using an L1 tagged with a retrotransposition indicator cassette, where the neo gene is interrupted with a self-splicing group I intron (Fig. 1B, L1.3neo ${ }^{I I I}$ ) (Esnault et al. 2002; Dewannieux et al. 2003), indicating that U6/L1 pseudogene formation probably does not depend on the association of L1 mRNA with the spliceosome. Although individual G418-resistant foci may contain multiple retrotransposition events, the efficiency of U6/L1 pseudogene formation seems to be elevated by at least an order of magnitude when compared with the number of U6/L1 pseudogenes observed in the HGWD. That said, we conclude that this experimental system faithfully reproduces retrotransposition events that occurred during the course of human genome evolution.

We next tested whether other small RNAs could form chimeric pseudogenes with L1. To accomplish this task, we designed two sets of primers for each snRNA and then performed PCR on DNA isolated from the same pools of G418-resistant foci used above (Methods). We identified a single U3/L1 pseudogene (Supplemental Fig. 3A; Supplemental Table 1), and the U3 sequence was 3 ' truncated at the same position identified in our in silico analysis. We also detected a single U6atac/L1 pseudogene (Supplemental Fig. 3B; Supplemental Table 1). In contrast, and consistent with our in silico analyses, we did not identify any U1/L1, U2/L1, U4/L1, U5/L1, hY1/L1, hY3/L1, or hY4/L1 pseudogenes in our assay (Supplemental Table 1). Thus, U6 snRNA, and to a much lesser extent U6atac and U3 small RNAs, can serve as template-switch substrates for the L1encoded reverse transcriptase.

\section{Processed pseudogene formation can occur by template choice}

The L1-encoded proteins can act in trans to promote the retrotransposition of other cellular RNAs (Esnault et al. 2000; Wei et al. 2001; Dewannieux et al. 2003; Ostertag et al. 2003; Bennett et al. 2004). We previously showed that RNA derived from an artificial construct (L1.3/ ORF1mneoI), which consists of the L1 5'UTR, ORF1, and that the mneoI retrotransposition indicator cassette could be used as a template for retrotransposition (Wei et al. 2001). Interestingly, the structure of L1.3/ORF1mneoI is similar to a repeated sequence present in all mammalian genomes (HAL1 [Half an L1], consensus sequence accessible from Repbase; Jurka et al. 2005). Thus, we examined the trans-mobilization of L1.3/ORF1mneoI RNA as a model to investigate the retrotransposition mechanism of HAL1like sequences.

To monitor ORF1mneoI retrotransposition, we exploited a trans-complementation assay that monitors the ability of a retrotransposition-competent "driver" L1 to trans-mobilize a "reporter" construct (Fig. 2A). Although the trans-complementation assay is relatively inefficient when compared with the cis-based retrotransposition assay, the assay yields hundreds of G418resistant foci and allows an experimental measure of pseudogene generation (Wei et al. 2001; Alisch et al. 2006). We demonstrated that efficient trans-complementation requires a functional ORF1p expressed from the reporter plasmid (Fig. 2A), and RNase protection experiments confirmed that wild-type ORF1mneoI RNA and its mutant derivatives were expressed in transfected cells at similar steady-state levels (Supplemental Fig. 4). These results indicate that ORF1mneoI retrotransposition requires the generation of a functional ribonucleoprotein (RNP) particle, which is consistent with our previous results using the cis-based retrotransposition assay (Kulpa and Moran 2005).

To determine whether ORF1mneoI pseudogene formation occurs by a template choice or template-switching mechanism, we introduced two nucleotides upstream of the poly(A) signal in our driver L1 (Fig. 2B, indicated by the star). If ORF1mneoI pseudogene formation occurs by template switching, the resultant retrotransposition events should contain the engineered SNPs at their respective 3 ' ends. In contrast, if ORF1mneoI pseudogene formation occurs by template choice, the resultant retrotransposition events should lack the engineered SNPs.

Twelve pools that contained $\sim 125$ (pools 1-9) or 450 (pools 10-12) G418-resistant foci were generated from HeLa cells that had been cotransfected with a driver L1 (pJM101/L1 $1_{\mathrm{RP}} \mathrm{SDM}$ No 
A

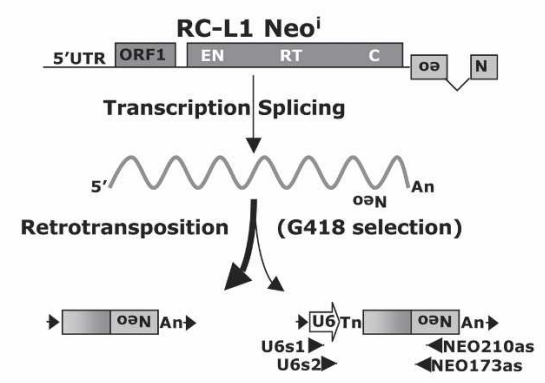

B
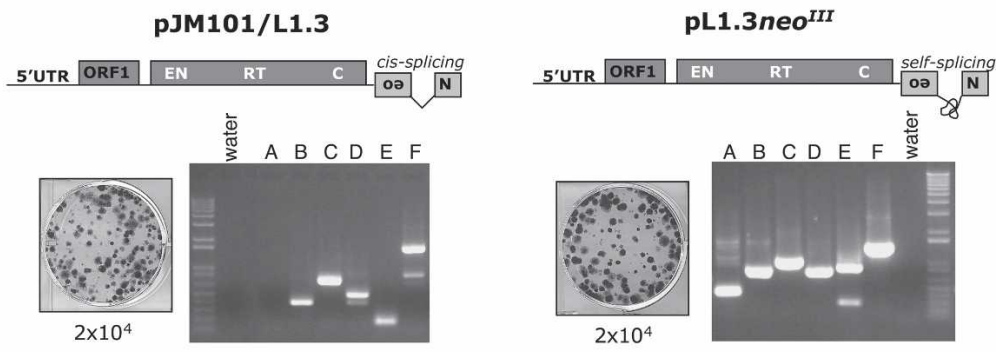

C

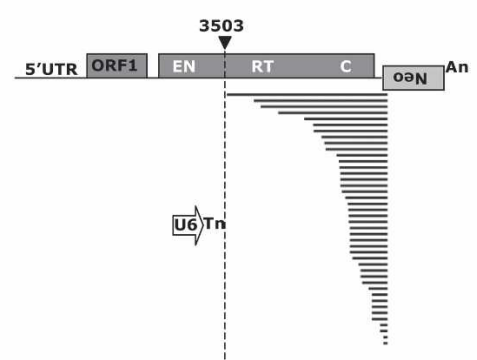

Figure 1. A cultured cell assay to detect U6/L1 pseudogenes. (A) Rationale of the assay. The 3' UTR of a retrotransposition-competent L1 (RC-L1) was tagged with a retrotransposition indicator cassette (light gray box labeled with a backward Neo). ORF1 and ORF2 are indicated by the dark-gray rectangles and the relative positions of the endonuclease (EN), reverse transcriptase (RT), and cysteine-rich domains (C) of ORF2 are indicated. Cartoons depicting the structures of the resultant retrotransposition events that confer $\mathrm{G} 418$ resistance $\left(\mathrm{G} 418^{\mathrm{R}}\right)$ to HeLa cells are shown at the bottom of the diagram. A 5'-truncated L1 insertion is shown at the left and a U6/L1 pseudogene is shown at the right. Target site duplications flanking the elements are represented as horizontal arrows. PCR primers used to detect U6/L1 pseudogenes are indicated below the U6/L1 schematic. (B) Results of the assay: Shown are schematic representations of two RC-L1s (pJM101/L1.3 and pL1.3neo $\left.{ }^{\prime \prime \prime}\right)$. pJM101/L1.3 contains our standard retrotransposition indicator cassette (Moran et al. 1996). pL1.3neo ${ }^{\prime \prime \prime}$ contains a retrotransposition indicator cassette disrupted by a self-splicing group I intron (Esnault et al. 2002; Dewannieux et al. 2003). Retrotransposition assays conducted by transfecting $2 \times 10^{4} \mathrm{HeLa}$ cells revealed that both L1s retrotranspose at similar efficiencies (Wei et al. 2000). Genomic DNAs derived from six independent pools of G418-resistant foci then were used as templates in nested PCR reactions with the primers noted in $A$ to detect the U6/L1 pseudogenes (lanes $A-F$ ). Molecular size standards are indicated on the gels (1-kb ladder plus from Invitrogen). (Water) PCR reactions conducted without the genomic DNA template. (C) Structures of the U6/L1 pseudogenes. Sequence analysis of the PCR products amplified in B confirmed the existence of the U6/L1 pseudogenes. All sequences contain the 3 ' terminus of U6 (depicted as an horizontal open arrow) and a variable 5' - truncated L1. A full-length RC-L1 with the spliced reporter cassette is represented at the top. The U6 sequence ends in 4-8 thymidine residues and is followed by a 5 '-truncated L1. The horizontal bold lines indicate the amount of the L1 sequence included in the pseudogene. The longest sequence extends to nucleotide 3503 of the L1. The numbering is based on the L1.2 reference sequence (Dombroski et al. 1991).

neo) and reporter (L1.3/ORF1mneoI) constructs (Fig. 2), and DNAs from each pool were then subjected to PCR to amplify the resultant retrotransposition events. The presence and/or absence of the engineered SNPs was then quantified using fluorescent primer-extension (FluPE) analysis (Fahy et al. 1997; BennettBaker et al. 2003). Serial dilution control experiments indicated that we could discriminate roughly one molecule in 250 (see Methods). However, despite examining genomic DNA from roughly 2500 G418-resistant foci, we never observed transfer of the diagnostic SNPs from the driver L1 to the resultant ORF1mneoI retrotransposition events (Fig. 2C). Thus, ORF1mneoI pseudogene formation predominantly occurs by a template choice mechanism. Although our experiments cannot formally exclude the possibility that template switching from the poly(A) tail of the driver L1 mRNA to the reporter mRNA generates some ORF1mneoI processed pseudogenes, our aforementioned studies of U6/L1 pseudogene formation make it very unlikely that all template-switching events would be confined to the poly(A) tails of the driver and reporter mRNAs.

Template choice and template switching can operate sequentially on the same RNA template

To determine whether the template choice and template-switching mechanisms could occur sequentially on the same RNA template, we combined the experimental strategies outlined in Figures 1 and 2 to test whether any L1.3/ ORF1mneoI-processed pseudogenes are conjoined to U6 snRNA-derived sequences (Fig. 3A). Consistent with data presented above, we found that approximately one in 20 ORF1mneoI pseudogenes contain U6 snRNA sequences at their $5^{\prime}$ ends (Fig. 3A). Sequence analysis of 23 independent PCR products indicated that the U6 sequences end in four to six thymidine residues, and then are followed by sequences derived from the ORF1mneoI expression construct (Fig. 3B; Supplemental Fig. 1). We further demonstrated that a synthetic mouse L1 (L1SM/ORF1mneoI) can generate U6/ pseudogenes (Supplemental Fig. 5), and that a U6/ORF1mneoI pseudogene exhibited hallmarks typical of an endonuclease-dependent L1 retrotransposition event (Fig. 3C), which is in agreement with our previously published data (Gilbert et al. 2005). Finally, our in silico analyses revealed that the HGWD contains at least $17 \mathrm{U6} /$ processed pseudogene chimeras, and we propose that these events were formed by template-choice/ template-switching mechanisms acting sequentially on the same RNA template in vivo.

U6/Alu pseudogenes are not formed efficiently in cultured cells

We next asked whether U6/Alu pseudogenes could be generated in HeLa cells. To accomplish this task, we cotransfected an Alu 
sequence tagged with a retrotransposition indicator cassette (pAluNF1neo ${ }^{I I}$ ) (Dewannieux et al. 2003) with either a retrotransposition-competent L1 lacking an indicator cassette (pJM101/ $\mathrm{L} 1_{\mathrm{RP}} \mathrm{No}$ neo) or an ORF2p expression construct (5'UTR ORF2 No neo; Supplemental Fig. 6A). The resultant G418-resistant foci were pooled in groups that ranged in size from 15 to 55 foci, and aliquots of genomic DNA from each pool were used as templates in nested PCR reactions using primers specific for U6 snRNA (U6s1 and U6s2) and the retrotransposition indicator cassette (210NEOas and 173NEOas). Although we could detect amplification products (Supplemental Fig. 6B), sequence analysis of 27 independent PCR amplicons indicated that they did not represent de novo U6/Alu pseudogenes, but instead were PCR artifacts (Supplemental Fig. 6C). Consistently, we determined that the majority (9/15) of U6/Alu pseudogenes identified in silico contained an A-rich linker between U6 and Alu and likely represent Alu insertions that occurred in the poly(A) tail of an existing U6 pseudogene. By comparison, at least four of the remaining six events might represent true U6/Alu-processed pseudogenes be-

\section{A}

\section{Driver L1}

\section{L1.3/ORF1mneoI}
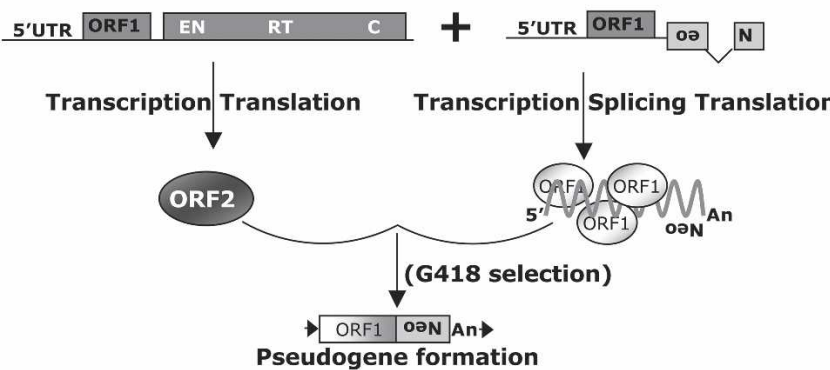

\begin{tabular}{|c|c|c|c|c|}
\hline \multirow{2}{*}{ Construct } & \multirow{2}{*}{ ORF1Status } & \multirow{2}{*}{$N$} & \multicolumn{2}{|c|}{ Number of G418R } \\
\cline { 4 - 5 } & & L1.3 No Neo & ORF2 No Neo \\
\hline L1.30RF1mneoI & Wild type & 2 & $252.5+/-20.5$ & $2031+/-36.1$ \\
\hline L1.3ORF1mneoI-AA & RR $_{261-262}$ AA & 2 & $2.5+/-0.7$ & $1.5+/-0.7$ \\
\hline L1.3ORF1mneoI-AKR & $R_{261} \mathrm{~K}$ & 2 & $5.5+/-0.7$ & $4+/-1.4$ \\
\hline
\end{tabular}

B

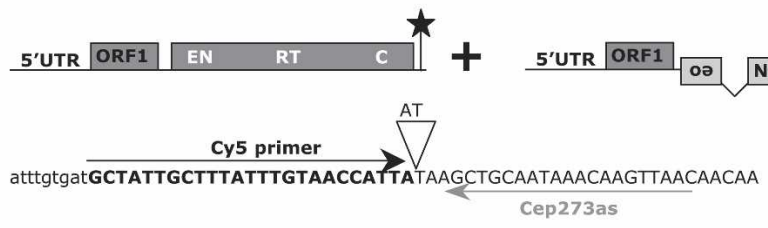

C

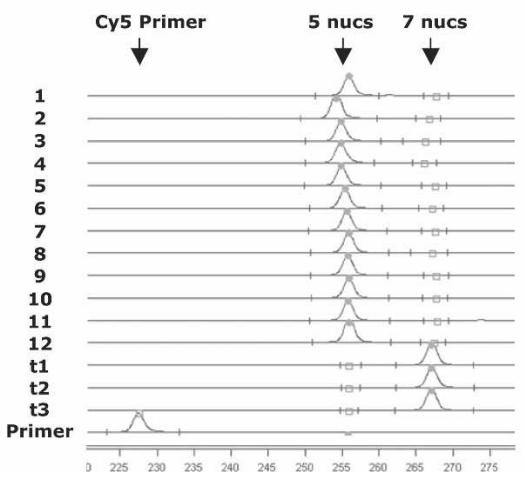

cause they are flanked by target-site duplications that range from 13 to $20 \mathrm{bp}$ in length.

\section{Discussion}

We have provided evidence that trans-mediated mobilization of cellular RNAs can occur via template-switching and/or templatechoice mechanisms. U6 snRNA (as well as U3 snoRNA and U6atac snRNA to a much lesser extent) likely use the L1 RT in the nucleus by a template-switching mechanism after the initiation of target-site primed reverse transcription (Fig. 4). However, it remains unclear whether these small RNAs are occasionally copackaged into the L1 ribonucleoprotein complex in the cytoplasm or whether they gain access to the L1 retrotransposition machinery in the nucleus. We further propose that ORF1mneoI (and likely other cellular RNAs) associate with the L1 reverse transcriptase in the cytoplasm by a template-choice mechanism and then complete the process of retrotransposition by target-site primed reverse transcription (Fig. 4). Indeed, the experimental demonstration of U6/ORF1mneoI pseudogenes and the identification of U6/processed pseudogene chimeras in the HGWD further suggests that the mechanisms of template choice and template switching are not necessarily mutually exclusive, and that both processes can act sequentially on the same RNA template.

Interestingly, Dewannieux et al. (2003) have reported that $A l u$ RNA also likely associates with the L1 reverse transcriptase in

Figure 2. ORF1p-dependent processed pseudogene formation occurs by template choice. (A) Rationale of the trans-complementation assay. A wild-type RC-L1 lacking the retrotransposition indicator cassette (pJM101/L1.3 No neo) or an ORF2 expression construct (ORF2 No neo) were cotransfected into HeLa cells with a construct consisting of the L1 5'UTR, ORF1, and the mneol retrotransposition indicator cassette (ORF1 mneol or mutant derivatives), which is a preferential substrate for trans-complementation (Wei et al. 2001). The CMV immediate early promoter augments the expression of both the driver and reporter constructs. G418-resistant foci will arise only if ORF1mneol RNA (i.e., the reporter $m R N A$ ) is trans-mobilized by ORF2p provided by the L1 lacking the indicator cassette (i.e., the driver L1). The inset Table indicates the names of the various reporter constructs (column 1), the status of ORF1 (column 2), the number of times the assay was conducted (column $3, \mathrm{~N}$ ), and the number of G418-resistant foci obtained using either driver L1 (column 4). ( $B, C$ ) Rationale and results of the fluorescent primer extension (FluPE) assay. The $3^{\prime}$ ends of the driver and reporter mRNAs were engineered so that the driver contains two extra nucleotides (indicated by the black star in the illustration of the driver construct and the inverted triangle in the depicted sequence). The driver and reporter constructs were cotransfected into HeLa cells and we isolated pools that contained 125-450 independent G418-resistant foci. Genomic DNA was extracted from the resultant pools and was subjected to PCR analysis using an oligonucleotide complementary to sequences in the indicator cassette (437NEOs) and another present just upstream of the poly(A) addition site in the vectors (Cep237as). Fluorescent Primer Extension (FluPE) then was conducted on the resultant PCR products using the Cy5 primer (black horizontal arrow). The arrows at bottom indicate the expected sizes of the Cy5 primer, the expected signal from the reporter construct (elongation of five nucleotides), and the expected signal from the driver construct (elongation of seven nucleotides). Retrotransposition events generated by template choice would be five nucleotides in size, whereas those formed via template switching would be seven nucleotides in size. The signals present in each pool (1-12) were five nucleotides in length, consistent with a template-choice mechanism for ORF1mneol pseudogene formation. Traces $\mathrm{t} 1, \mathrm{t} 2$, and $\mathrm{t} 3$ represent positive control FluPE reactions performed on genomic DNA from cells transfected with an RC-L1 containing both the 2-bp insertion and the retrotransposition indicator cassette (pJM101/L1 $\left.1_{R P} S D M\right)$. All experiments were repeated at least three times with the same results. The $X$-axis represents the running time of the reaction (minutes). 
the cytoplasm and that its retrotransposition does not require the ORF1-encoded protein. However, we did not observe a high efficiency of U6/Alu pseudogene formation in cultured cells. Thus, these data suggest that ORF1p enhances the retrotransposition of U6 snRNA. Our results also indicate that U6 snRNA is more efficiently dispersed throughout the genome when compared with

\section{A}
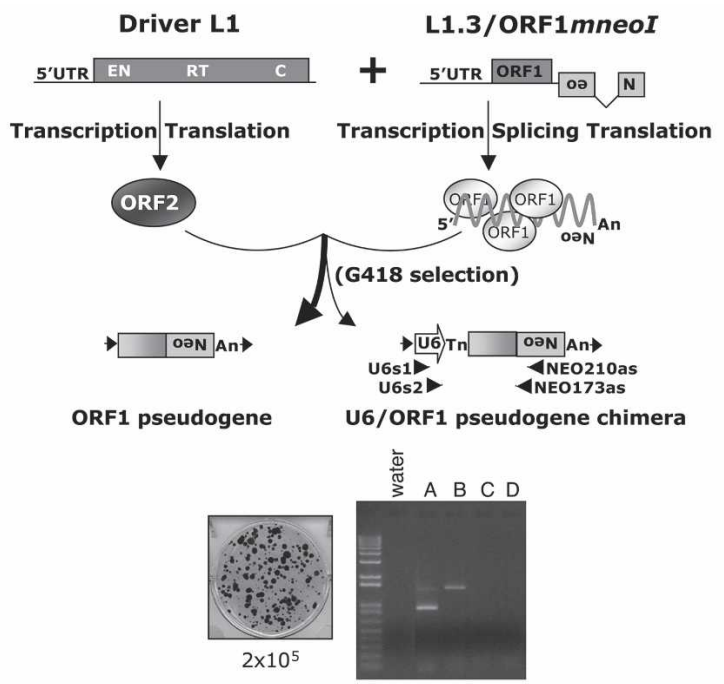

5'UTR ORF2 + L1.3/ORF1mneoI

B

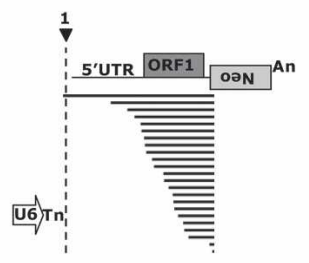

C aaccettcttAGAAGGCCTCTG \begin{tabular}{|l|l|l|}
\hline & OəN \\
\hline
\end{tabular}

Figure 3. Template choice and template switching are not mutually exclusive. (A) Rationale and results of the assay. The transcomplementation assay is described in Figure 2. Retrotransposition assays were conducted by transfecting $2 \times 10^{5}$ HeLa cells with the driver $\left(5^{\prime}\right.$ UTR ORF2 No neo) and reporter (L1.3/ORF1mneol) plasmids. Genomic DNAs from pools of G418-resistant foci were subjected to individual nested PCR reactions using the indicated primers (U6s1-NEO210as and U6s2-NEO173as) to detect U6/ORF1mneol pseudogenes. (B) Results of the assay. Sequence analysis of 23 PCR products confirmed the existence of the U6/ORF1mneol pseudogenes. A full-length ORF1mneol mRNA is represented at top. The $\mathrm{U} 6$ sequence ends in four to six thymidine residues and is followed by ORF1 mneol RNA. The horizontal bold lines indicated the amount of the L1 included in the pseudogene. The longest U6/ORF1 mneol extends beyond the first nucleotide of ORF1 mneol RNA, indicating that the transcript was initiated from the CMV promoter upstream of the reporter gene. (C) The structure of a U6/ORF1mneol pseudogene. The characterization of a single U6/ORF1 mneol pseudogene by inverse PCR revealed the hallmarks of L1 retrotransposition. Top bold characters on each side of the pseudogene represent the target site duplication. Lowercase characters represent the flanking genomic sequence. The complement of the L1 endonuclease cleavage recognition site $\left(5^{\prime}-T T C T / A\right)$ is underlined. The numbering is based on the L1.2 reference sequence (Dombroski et al. 1991).

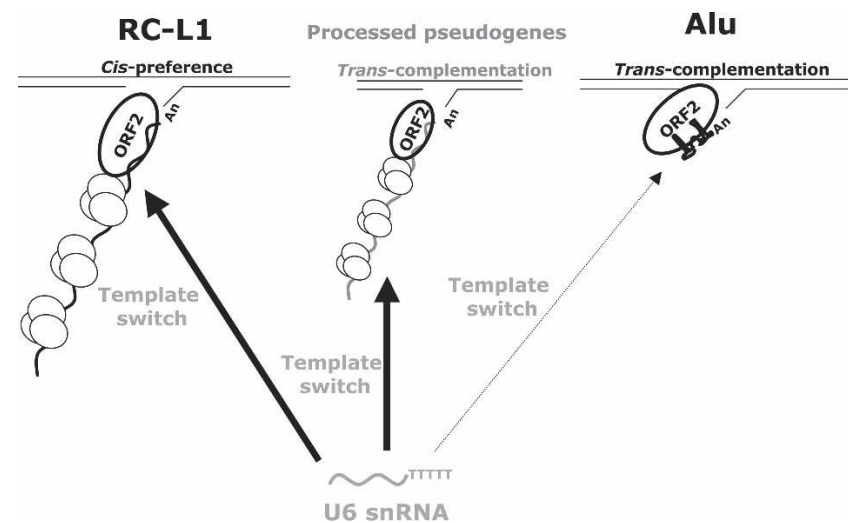

Figure 4. Trans-mobilization of non-L1 mRNAs by the L1 retrotransposition machinery. The L1-encoded proteins preferentially bind to the RNA that encoded them in cis to promote their retrotransposition by targetsite primed reverse transcription (left). Cellular RNAs (e.g., ORF1mneol, middle; or Alu RNA, right) can occasionally use the L1 RT to mediate their mobility in trans. During target-site primed reverse transcription a template switch from L1 mRNA to U6 snRNA can lead to the formation U6/L1 pseudogenes. The data indicate that U6/ORF1 mneol pseudogenes can be generated by sequential template choice and template-switching mechanisms. However, U6/Alu pseudogenes are not efficiently generated in our experimental system, leading us to propose that ORF1p promotes efficient U6 snRNA retrotransposition. ORF1p molecules are depicted as small ovals associated in trimers (Martin et al. 2003). ORF2p is drawn as a large bolded oval. L1, ORF1 mneol, and Alu mRNA that are being reverse transcribed by target-site primed reverse transcription are indicated. U6 snRNA is indicated in gray toward the bottom of the figure. Whether U6 snRNA gains access to the L1 retrotransposition machinery in the cytoplasm or nucleus remains unknown. As proposed, the Alu RNP complex is shown without ORF1p (Dewannieux et al. 2003). However, it remains possible that some Alu RNA is associated with ORF1p in vivo.

other abundant snRNAs. Why U6 snRNA seems to serve as a preferential substrate for the L1 reverse transcriptase requires further study. However, because we detect efficient U6/L1 chimeric pseudogene formation with "driver" L1s that are equipped with a retrotransposition indicator cassette containing a self-splicing group I intron or a codon optimized "synthetic" mouse L1, we hypothesize that U6 snRNA does not necessarily gain access to the $\mathrm{L} 1$ retrotransposition machinery because of its association with the spliceosome.

In closing, our data highlight mechanisms by which the LINE-1 encoded proteins can mobilize non-L1 RNAs and strongly suggest that U6 snRNA can still undergo retrotransposition in the human genome. The copy number of U6/L1 pseudogenes and the finding that U6 snRNA serves as a preferential substrate for LINE-1-mediated retrotransposition when compared with other snRNAs would suggest that they represent a new SINE family. However, since a functional U6 snRNA gene contains a proximal sequence element located upstream of its transcription-initiation site (Kunkel and Pederson 1988; Lobo and Hernandez 1989), most retrotransposed U6/L1 chimeras should lack promoter sequences and will be unable to undergo subsequent rounds of retrotransposition and will be "dead on arrival." It remains possible that some retrotransposed U6 snRNA sequences may integrate near a RNA pol III promoter, leading to the generation of a transcriptionally active "founder" locus. Indeed, such copies may represent "stealth" drivers that contribute to the ongoing interspersion of U6 elements in a manner analogous to that described previously for Alu elements (Han et al. 2005). Clearly, future experiments are needed to address this interesting possibility. 


\section{Methods}

\section{Oligonucleotides}

173NEOs: 5'-CAAGAAGGCGATAGAAGGCGATG-3'; 437NEOs: 5'-GAGCCCCTGATGCTCTTCGTCC-3'; 664NEOs: 5' -CCC TTCCCGCTTCAGTGACAA-3'; 1808NEOs: 5'-GCGTGCAATCC ATCTTGTTCAATG-3'; 173NEOas: 5'-CATCGCCTTCTATCGCC TTCTTG-3'; 210NEOas: 5'-GACCGCTTCCTCGTGCTTTACG-3'; U1s1: 5'-TGTGGGAAACTCGACTGC-3'; U1s2: 5'-GTGGTAG TGGGGGACTGCG-3'; U2s1: 5'-GGAAATAGGAGATGGAATAG3'; U2s2: 5'-GCTTGCTCCGTCCACTCC-3'; U3s1: 5'-CTATAC TTTCAGGGATCATTTC-3'; U3s2: 5'-GTTCGTTACTAGAGAAG TTTC-3'; U4s1: 5'-CAATACCCCGCCATGACGAC-3'; U4s2: 5'GAAATATAGTCGGCATTGGC-3'; U5s1: 5'-CCGTGGAGAGGAA CAAC-3'; U5s2: 5'-CTGAGTCTTAACCCAATT-3'; U6s1: 5' ACAGAGAAGATTAGCATGGC-3'; U6s2: 5'-CCCTGCGCAAG GATGAC-3'; U6atac_s1: 5'-TGACAAGGATGGAAGAGGCCCT-3'; U6atac_s2: 5'-CTGACAACACGCATACGGTTAAG-3'; hY1s1: 5' GTCAGTTACAGATCGAAC-3'; hY1s2: 5'-CTTGTTCTACTC TTTCCCC-3'; hY3s1: 5' -CAACTAATTGATCACAAC-3'; hY3s2: 5'GTTACAGATTTCTTTGTTCCTTC-3'; hY4s1: 5' -TATTAA CATTAGTGTCACTAAAG-3'; hY4s2: 5'-GGTATACAACCC CCCACTGCT-3'; U6as1: 5'-GCCATGCTAATCTTCTCTGT-3'; U6as 2: 5'-GTCATCCTTGCGCAGGG-3'; JA10: 5'-GAT CTCGGCCGAAAAGCGCAATATTCGGGT-3'; JB3165: 5' AATTAACCCTCACTAAAGGGCAGGTTGACGCAAATGGGCGG TAGGCGTGTACGG-3'; JB3168: 5'-TAATACGACTCACTATAGGG CAGCGGGCAGTTCGGTTTCAGGCAGGTCTTGC-3'; JB3167: 5' AATAACCCTCACTAAAGGGCAGCCAGCGTCTTGTCAT TGGCGAATTCGAACACGC-3'; SDM295PacIs: (5'-GCTTTATT TGTAACCATTAATTAAG CTGCAATAAACAAGTTAAC- ${ }^{\prime}{ }^{\prime}$ ); SDM295PacIas: (5'-GTTAACTTGTTTATTGCAGCTTAATT AATGGTTACAAATAAAGC-3'); Cep273as: 5'-GTTAACTTGTTTAT TGCAGC-3'.

\section{Recombinant DNA plasmids}

The following plasmids contain the indicated fragments cloned in pCEP4 (Invitrogen), unless otherwise indicated. Cloning strategies are available upon request.

pJM101/L1.3 contains a 8.2-kb NotI-BamHI fragment containing a full-length copy of the L1.3 element and the mneoI indicator cassette (Dombroski et al. 1993; Sassaman et al. 1997).

pJM101/L1.3 No neo contains a 6.0-kb NotI-BamHI fragment containing a full-length copy of the L1.3 element (Wei et al. 2001).

pJM101/L1 $1_{\mathrm{RP}}$ contains a 8.2-kb NotI-BamHI fragment containing a full-length copy of the $\mathrm{L} 1_{\mathrm{RP}}$ element and the mneoI indicator cassette (Kimberland et al. 1999).

pJM101/L1 $1_{\mathrm{RP}}$ No neo contains a 6.0-kb NotI-BamHI fragment containing a full-length copy of the $\mathrm{L} 1_{\mathrm{RP}}$ element (Wei et al. 2001).

L1.3/ORF1mneoI contains a 3.8-kb NotI-BamHI fragment containing the L1.3 5'UTR, L1.3 ORF1, and the mneoI indicator cassette (Wei et al. 2001).

L1.3/ORF1mneoI-AAA was created by swapping the NotI-AgeI fragment from L1.3 ORF1mneoI with the corresponding fragment from pDK105 (Kulpa and Moran 2005). It contains two arginine-alanine mutations in ORF1 (RR261-262AA).

L1.3/ORF1mneoI-AKR was created by swapping the NotI-AgeI fragment from L1.3 ORF1mneoI with the corresponding fragment from pDK107 (Kulpa and Moran 2005). It contains an arginine-lysine mutation in ORF1 (R261K).

$p J M 101 / L 1_{\mathrm{RP}}$ No neo $S D M$ is a derivative of $\mathrm{pJM} 101 / \mathrm{L} 1_{\mathrm{RP}}$ No neo that contains a two-base insertion (AT) $26 \mathrm{bp}$ upstream the
SV40 poly(A) site present in pCEP4. The insertion creates a PacI restriction site. Site-directed mutagenesis was performed using the QuickChange XL Site-Directed Mutagenesis Kit (Stratagene) using the SDM295PacIs and SDM295PacIas primers. $p J M 101 / L 1_{\mathrm{RP}} S D M$ is a derivative of $\mathrm{pJM} 101 / \mathrm{L} 1_{\mathrm{RP}}$ No neo SDM that contains the mneoI indicator cassette.

pCEPTG $_{f} 21$ contains a 8.8-kb NotI-BamHI fragment containing a full-length mouse $\mathrm{TG}_{\mathrm{f}} 21 \mathrm{~L} 1$ element and the mneoI indicator cassette (Goodier et al. 2001).

pCEPL1SM contains a 9.5-kb NotI-BamHI fragment containing a full-length synthetic mouse $\mathrm{L} 1\left(\mathrm{~L} 1_{\mathrm{spa}}\right)$ and the mneoI indicator cassette. The sequence of both ORFs was codon optimized (Han and Boeke 2004).

pL1SM/ORF1mneoI contains a 4.1-kb NotI-BamHI fragment containing the L1SM 5'UTR, the L1SM ORF1, and the mneoI indicator cassette.

pCEP 5'UTR ORF2 No Neo contains a 5.1-kb NotI-BamHI fragment containing the L1.3 5'UTR and L1.3 ORF2 (Alisch et al. 2006).

pAluNF1-neo ${ }^{I I I}$ has been described previously (Dewannieux et al. 2003). It contains a $2.1-\mathrm{kb}$ fragment containing a 7SL promoter, a copy of the NF1 Alu (Wallace et al. 1991), the neo ${ }^{I I I}$ self-splicing indicator cassette (Esnault et al. 2002), a 44-bp poly(A) tail, and a 7SL transcription termination sequence cloned in pUC19 (Dewannieux et al. 2003).

pL1.3neo ${ }^{I I I}$ contains a 8.0-kb NotI-BamHI fragment containing a full-length copy of the L1.3 element and the $n e o^{I I I}$ self-splicing indicator cassette (Dombroski et al. 1993; Sassaman et al. 1997; Esnault et al. 2002).

\section{In silico analysis}

Sequences of the small nuclear RNA genes used to perform BLAST search on Ensembl v40 and v41 (August and October 2006; http://www.ensembl.org/Homo_sapiens/blastview) are as follows: U1 accession no. J00318, U2 accession no. K02227, U3 accession no. AF020534, U4 accession no. M15957, U5 accession no. X04215, U6 accession no. M14486, U11 accession no. X58716, U12 accession no. J04119, U4atac accession no. U62822, and U6atac accession no. U62823. We limited our analysis to the sequences having an E value $\leq 5 \times 10^{-27}$ corresponding to a U6 full-length sequence (107 bp) with $<10 \%$ divergence from the query sequence.

\section{DNA preparation}

Plasmid DNAs were purified using QIAGEN midi-prep columns (QIAGEN). HeLa genomic DNA was isolated using either the Blood and Cell Midi-prep kit (QIAGEN) or the Cell and Tissue DNA isolation kit (Puregene, Gentra).

\section{Cell culture}

HeLa cells were grown at $37^{\circ} \mathrm{C}$ in an atmosphere containing $7 \%$ carbon dioxide and 100\% humidity in Dulbecco's modified Eagle medium (DMEM) lacking pyruvate (GIBCO BRL). DMEM was supplemented with $10 \%$ fetal bovine calf serum and $1 \times$ Penicillin-Streptomycin-L-glutamine (a $100 \times$ stock is sold by GIBCO BRL). Cell passage and cloning (by either limiting dilution or colony lifting) was performed using standard techniques.

\section{Cis and trans retrotransposition assays}

Both assays have been described previously (Moran et al. 1996; Wei et al. 2000, 2001; Bogerd et al. 2006). Briefly, in the cisretrotransposition assay $2 \times 10^{4}$ and/or $2 \times 10^{3}$ HeLa cells were 
plated in duplicate 6-well tissue culture dishes. After 12-14 h, one set of 6-well plates was cotransfected with equal amounts of a reporter plasmid (humanized Renilla green fluorescent protein [hr-EGFP; Stratagene]) and a L1 tagged with the mneoI indicator cassette. The other set of 6-well plates was transfected with only the $\mathrm{L} 1$ construct. We routinely used $3 \mu \mathrm{L}$ of Fugene 6 transfection reagent (Roche Molecular Biochemical) and $1.0 \mu \mathrm{g}$ of DNA per well. Seventy-two hours post-transfection, the HeLa cells in one set of 6-well tissue culture plates was trypsinized and subjected to flow cytometry (Ostertag et al. 2000; Wei et al. 2000). The percentage of green fluorescent (GFP) cells was used to determine the transfection efficiency of each sample. The remaining set of 6-well plates was subjected to G418 selection $(400 \mu \mathrm{g} / \mathrm{mL})$. After $12 \mathrm{~d}$, pools of G418-resistant cells were collected from three wells and genomic DNA was extracted. The remaining three wells were washed in $1 \times$ phosphate-buffered saline (PBS), fixed by incubation in FIX solution (2\% formaldehyde [of a $37 \%$ stock solution in water], $0.2 \%$ glutaraldehyde, $1 \times \mathrm{PBS}$ ) at $4^{\circ} \mathrm{C}$ for $30 \mathrm{~min}$. The fixed cells were stained with either $0.1 \%$ Brilliant Blue or $0.1 \%$ Crystal Violet at room temperature overnight, washed with water, and then manually counted. The retrotransposition efficiency is expressed as the number of G418-resistant foci/the number of transfected (EGFP-positive) cells. In the transretrotranspositon assay, $2 \times 10^{5}$ and/or $2 \times 10^{4} \mathrm{HeLa}$ cells were plated in 6-well tissue culture dishes. Approximately 12-14 h after plating, cells were cotransfected with equal amounts of a "reporter plasmid" (e.g., L1.3/ORF1mneoI, L1SM/ORF1mneoI, or Alu-NF1 neo ${ }^{I I}$ ) and a "driver" L1 that lacked the mneoI indicator cassette (e.g., JM101/L1 $1_{\mathrm{RP}}$ No neo or 5'UTR ORF2 No neo). Transfection conditions, transfection efficiency calculations, G418 selection, and pool harvesting was preformed as described above. When a mutant L1.3/ORF1mneoI construct was assayed in the trans-retrotranspositon assay, $6 \times 10^{6} \mathrm{HeLa}$ cells were plated in a $\mathrm{T}-175 \mathrm{~cm}^{2}$ flask and transfected with equal amounts of a "driver" L1 and "reporter" plasmid using established protocols (Wei et al. $2000,2001)$. Retrotransposition assays were performed as described above.

\section{RNase protection assays}

HeLa cells were plated in T-175 $\mathrm{cm}^{2}$ flasks at a density of $6 \times 10^{6}$ cells/flask and were transfected with $30 \mu \mathrm{g}$ of the ORF1mneoI expression plasmids using $90 \mu \mathrm{L}$ of the Fugene 6 transfection reagent (Roche Molecular Biochemicals) (Wei et al. 2000). Cells were harvested $72 \mathrm{~h}$ post-transfection and total RNA was prepared using the TRIzol reagent (Invitrogen). RNA samples were treated with $100 \mathrm{U}$ of RNase-free DNase I (Roche Molecular Biochemicals) for $1 \mathrm{~h}$ at $37^{\circ} \mathrm{C}$, extracted with acid-equilibrated phenolchloroform, and precipitated with $2 \mathrm{Vol}$ of $100 \%$ ethanol. PCR products containing T7 promoter sequences were used as templates for in vitro transcription, which was carried out using T7 RNA polymerase in the presence of $\left[\alpha-{ }^{32} \mathrm{P}\right] \mathrm{UTP}$ using the Maxiscript in vitro transcription kit (Ambion). The primers JB3169 and JA10 were used to generate the L1 probes. JB3168 and JB3167 were used to generate the $h y g$ probe. We used a Hybaid Thermocycler programmed as follows: One cycle of $95^{\circ} \mathrm{C}$ for 10 min, $55^{\circ} \mathrm{C}$ for $7 \mathrm{~min}, 72^{\circ} \mathrm{C}$ for $1 \mathrm{~min}$, followed by 30 cycles of $95^{\circ} \mathrm{C}$ for $30 \mathrm{sec}, 55^{\circ} \mathrm{C}$ for $30 \mathrm{sec}, 72^{\circ} \mathrm{C}$ for $1 \mathrm{~min}$, followed by a final extension step of $72^{\circ} \mathrm{C}$ for $10 \mathrm{~min}$. RNase protection assays were performed using the RPA III kit (Ambion). Briefly, $30 \mu \mathrm{g}$ of total RNA was hybridized to gel-purified probe $\left(1 \times 10^{5} \mathrm{cpm} /\right.$ reaction) at $42^{\circ} \mathrm{C}$ overnight. The reactions were then digested in a mixture of RNase $\mathrm{A}(0.375 \mathrm{U})$ and RNase $\mathrm{T} 1(15 \mathrm{U})$ at $37^{\circ} \mathrm{C}$ for $1 \mathrm{~h}$, precipitated, and resolved on a $6 \%$ denaturing polyacrylamide gel.

\section{Fluorescent primer extension}

Genomic DNAs from pools of G418-resistant colonies generated from the trans-complementation assay were used as templates in PCR reactions with oligonucleotides specific to the indicator cassette (437NEOs) and a sequence in the engineered $\mathrm{L} 1$ that was located upstream of the SV40 poly(A) site in the pCEP4 vector (cep273as). PCR was performed as follows: one cycle of $94^{\circ} \mathrm{C}$ for $2 \mathrm{~min}$, followed by 30 cycles of $94^{\circ} \mathrm{C}$ for $30 \mathrm{sec}, 60^{\circ} \mathrm{C}$ for $30 \mathrm{sec}$, and $72^{\circ} \mathrm{C}$ for $1 \mathrm{~min}$, followed by a final extension at $72^{\circ} \mathrm{C}$ for 10 min. We used the PCR product to conduct Fluorescent Primer Extension (FluPE) reactions. Primer extension was performed in a volume of $8 \mu \mathrm{L}$ containing $100 \mathrm{fmol}$ of the PCR product, 100 fmol of the Cy5 primer, $25 \mu \mathrm{M}$ each dNTP (we used ddCTP to limit the extension after the nucleotide polymorphism), $2 \mathrm{mM}$ $\mathrm{MgCl}_{2}, 10 \mathrm{mM}$ Tris- $\mathrm{HCl}$ (pH 8.8), $10 \mathrm{mM} \mathrm{KCl,} \mathrm{0,002 \%} \mathrm{Tween-20,}$ and $0.64 \mathrm{U}$ of Thermo sequenase (Amersham Pharmacia Biotech). Extension reactions were cycled as follows: one cycle of $94^{\circ} \mathrm{C}$ for $2 \mathrm{~min}$, followed by 25 cycles of $55^{\circ} \mathrm{C}$ for $40 \mathrm{sec}$ and $94^{\circ}$ for $30 \mathrm{sec}$. The products were resolved on a 19\% denaturing polyacrylamide gel using the ALFexpress II (Amersham Pharmacia Biotech) automated sequencing system. The relative peak fluorescence signal was measured for each extension product using the AlleleLinks software package (v1.01) (Bennett-Baker et al. 2003). To calculate the sensitivity of the reaction, PCR was used to amplify DNA fragments containing or lacking the two-nucleotide polymorphism. The resultant DNA fragments were quantified and serially diluted to produce "reporter" (ORF1mneoI allele) to "driver" (JM101/L1 $1_{\mathrm{RP}} \mathrm{SDM}$ allele) ratio mixtures of 1:1, 1:5, 1:10, $1: 50,1: 100,1: 250,1: 500$, and 1:1000, respectively. Each mixture was used as a template in the primer-extension assay. We could reproducibly detect a signal in the 1:250 dilution sample.

\section{Nested PCR amplification}

Chimeric pseudogenes (snRNA/L1 and U6/Alu) were amplified using a nested PCR protocol on genomic DNAs derived from pools of G418-resistant colonies (see above). First-round PCR reactions were conducted in a volume of $50 \mu \mathrm{L}$ using oligonucleotides specific to the snRNA (oligonucleotides s1) and NEO gene (210NEOas) using $200 \mathrm{ng}$ of genomic DNA as a template. Secondround PCR reactions were performed using $4 \mu \mathrm{L}$ of the first-round PCR as a template with the $s 2$ primer and 173 NEOas oligonucleotide primers. All PCR reactions were performed using High Fidelity Taq (Roche Molecular Biochemical) in an express thermal cycler (Hybaid) using the following cycling conditions: one cycle at $95^{\circ} \mathrm{C}$ for $2 \mathrm{~min}$, followed by 30 cycles at $94^{\circ} \mathrm{C}$ for $15 \mathrm{sec}, 55^{\circ} \mathrm{C}$ for $20 \mathrm{sec}$, and $72^{\circ} \mathrm{C}$ for $3 \mathrm{~min}$, followed by a final extension cycle of $72^{\circ} \mathrm{C}$ for $10 \mathrm{~min}$. The PCR products from the second amplification were purified using the QIAquick PCR purification kit (QIAGEN) and sequenced on an Applied Biosystems DNA sequencer (ABI 377) at the University of Michigan Core facilities or at the IGH. Some PCR products were cloned in pGEMT-Easy (Promega) prior to sequencing.

\section{Inverse PCR}

Inverse PCR was performed essentially as described previously (Morrish et al. 2002). Briefly, $5 \mu \mathrm{g}$ of genomic DNA derived from G418-resistant colonies was digested to completion with SspI (New England Biolabs) in a total reaction volume of $50 \mu \mathrm{L}$. Heating at $65^{\circ} \mathrm{C}$ for $30 \mathrm{~min}$ stopped the reactions. The restricted DNA was ligated using T4 DNA ligase (3200 U; New England Biolabs) in a volume of $600 \mu \mathrm{L}$ at $16^{\circ} \mathrm{C}$ for at least $16 \mathrm{~h}$. The ligated DNA was precipitated with ethanol and dissolved in $40 \mu \mathrm{L}$ of distilled water. Two microliters of DNA were used in the primary PCR reaction in a $50-\mu \mathrm{L}$ reaction volume containing a $20-\mathrm{nM}$ concen- 
tration of each dNTP, 10 pmol of primers U6as2 and 664NEOs, $1 \times$ buffer 3, and $2.5 \mathrm{U}$ of enzyme mix in the Expand Long Template PCR system (Roche Molecular Biochemicals). We used a Hybaid Thermocycler programmed as follows: one cycle of $95^{\circ} \mathrm{C}$ for $2 \mathrm{~min}$, followed by 30 cycles of $94^{\circ} \mathrm{C}$ for $10 \mathrm{sec}, 63^{\circ} \mathrm{C}$ for 30 sec, and $68^{\circ} \mathrm{C}$ for $15 \mathrm{~min}$, followed by a final extension step at $68^{\circ} \mathrm{C}$ for $30 \mathrm{~min}$. The resultant products $(1 \mu \mathrm{L})$ then were used in a secondary PCR reaction using the same conditions, except that we used primers U6as1 and 1808NEOs. PCR products from the second amplification were purified with QIAquick PCR purification kit (QIAGEN) and sequenced. Sequence flanking the insertion was used as probe in BLAT search (http://genome. cse.ucsc.edu) to identify the preintegration site in the HGWD (Kent 2002).

\section{Acknowledgments}

We thank members of the IGH and the University of Michigan Sequencing Core for DNA sequencing assistance. We thank John Goodier for providing the $\mathrm{TG}_{\mathrm{f}} 21$ "natural" mouse L1, Jef Boeke for providing the synthetic mouse L1 element, and Thierry Heidmann for providing the Alu expression cassette. We thank Matthew Pratt-Hyatt, Laure Boizeau, Aurélie Chanut, and Oussama Meziane for their excellent technical assistance. We thank Lee Lawton and David Burke for assistance with the FluPE experiments. This work was supported in part by grants to N.G. from the Centre National de Recherche Scientifique and the Agence Nationale de la Recherche (JC05 63089). A.J.D. was the recipient of a fellowship from the French government (Ministère de l'Enseignement Supérieur et de la Recherche). J.V.M. was supported in part by the National Institutes of Health (GM60518). J.L.G.P. was supported in part by a MEC/Fulbright postdoctoral grant EX-2003-0881 (MEC, Spain).

\section{References}

Alisch, R.S., Garcia-Perez, J.L., Muotri, A.R., Gage, F.H., and Moran, J.V. 2006. Unconventional translation of mammalian LINE-1 retrotransposons. Genes \& Dev. 20: 210-224.

Bennett, E.A., Coleman, L.E., Tsui, C., Pittard, W.S., and Devine, S.E. 2004. Natural genetic variation caused by transposable elements in humans. Genetics 168: 933-951.

Bennett-Baker, P.E., Wilkowski, J., and Burke, D.T. 2003. Age-associated activation of epigenetically repressed genes in the mouse. Genetic 165: 2055-2062

Bogerd, H.P., Wiegand, H.L., Hulme, A.E., Garcia-Perez, J.L., O'Shea, K.S., Moran, J.V., and Cullen, B.R. 2006. Cellular inhibitors of long interspersed element 1 and Alu retrotransposition. Proc. Natl. Acad. Sci. 103: 8780-8785.

Brosius, J. 1991. Retroposons-seeds of evolution. Science 251: 753.

Brosius, J. 1999. Genomes were forged by massive bombardments with retroelements and retrosequences. Genetica 107: 209-238.

Brouha, B., Schustak, J., Badge, R.M., Lutz-Prigge, S., Farley, A.H., Moran, J.V., and Kazazian Jr., H.H. 2003. Hot L1s account for the bulk of retrotransposition in the human population. Proc. Natl. Acad. Sci. 100: 5280-5285

Buzdin, A., Ustyugova, S., Gogvadze, E., Vinogradova, T., Lebedev, Y., and Sverdlov, E. 2002. A new family of chimeric retrotranscripts formed by a full copy of U6 small nuclear RNA fused to the $3^{\prime}$ terminus of 11. Genomics 80: 402-406.

Buzdin, A., Gogvadze, E., Kovalskaya, E., Volchkov, P., Ustyugova, S. Illarionova, A., Fushan, A., Vinogradova, T., and Sverdlov, E. 2003. The human genome contains many types of chimeric retrogenes generated through in vivo RNA recombination. Nucleic Acids Res. 31: $4385-4390$.

Cost, G.J., Feng, Q., Jacquier, A., and Boeke, J.D. 2002. Human L1 element target-primed reverse transcription in vitro. $E M B O \mathrm{~J}$. 21: $5899-5910$.

Denison, R.A., Van Arsdell, S.W., Bernstein, L.B., and Weiner, A.M. 1981. Abundant psuedogenes for small nuclear RNAs are dispersed in the human genome. Proc. Natl. Acad. Sci. 78: 810-814.
Dewannieux, M., Esnault, C., and Heidmann, T. 2003. LINE-mediated retrotransposition of marked Alu sequences. Nat. Genet. 35: 41-48.

Dombroski, B.A., Mathias, S.L., Nanthakumar, E., Scott, A.F., and Kazazian Jr., H.H. 1991. Isolation of an active human transposable element. Science 254: 1805-1808.

Dombroski, B.A., Scott, A.F., and Kazazian Jr., H.H. 1993. Two additional potential retrotransposons isolated from a human L1 subfamily that contains an active retrotransposable element. Proc. Natl. Acad. Sci. 90: 6513-6517.

Ergun, S., Buschmann, C., Heukeshoven, J., Dammann, K., Schnieders, F., Lauke, H., Chalajour, F., Kilic, N., Stratling, W.H., and Schumann, G.G. 2004. Cell type-specific expression of LINE-1 open reading frames 1 and 2 in fetal and adult human tissues. J. Biol. Chem. 279: 27753-27763.

Esnault, C., Maestre, J., and Heidmann, T. 2000. Human LINE retrotransposons generate processed pseudogenes. Nat. Genet. 24: 363-367.

Esnault, C., Casella, J.F., and Heidmann, T. 2002. A Tetrahymena thermophila ribozyme-based indicator gene to detect transposition of marked retroelements in mammalian cells. Nucleic Acids Res. 30: e49.

Fahy, E., Nazarbaghi, R., Zomorrodi, M., Herrnstadt, C., Parker, W.D., Davis, R.E., and Ghosh, S.S. 1997. Multiplex fluorescence-based primer extension method for quantitative mutation analysis of mitochondrial DNA and its diagnostic application for Alzheimer's disease. Nucleic Acids Res. 25: 3102-3109.

Feng, Q., Moran, J.V., Kazazian Jr., H.H., and Boeke, J.D. 1996. Human L1 retrotransposon encodes a conserved endonuclease required for retrotransposition. Cell 87: 905-916.

Freeman, J.D., Goodchild, N.L., and Mager, D.L. 1994. A modified indicator gene for selection of retrotransposition events in mammalian cells. Biotechniques 17: 46, 48-49, 52.

Gilbert, N., Lutz, S., Morrish, T.A., and Moran, J.V. 2005. Multiple fates of 11 retrotransposition intermediates in cultured human cells. Mol. Cell. Biol. 25: 7780-7795.

Gogvadze, E.V., Buzdin, A.A., and Sverdlov, E.D. 2005. [Multiple template switches on LINE-directed reverse transcription: The most probable formation mechanism for the double and triple chimeric retroelements in mammals]. Bioorg. Khim. 31: 82-89.

Goodier, J.L., Ostertag, E.M., Du, K., and Kazazian Jr., H.H. 2001. A novel active L1 retrotransposon subfamily in the mouse. Genome Res. 11: $1677-1685$

Han, J.S. and Boeke, J.D. 2004. A highly active synthetic mammalian retrotransposon. Nature 429: 314-318

Han, K., Xing, J., Wang, H., Hedges, D.J., Garber, R.K., Cordaux, R., and Batzer, M.A. 2005. Under the genomic radar: The stealth model of Alu amplification. Genome Res. 15: 655-664.

Hohjoh, H. and Singer, M.F. 1996. Cytoplasmic ribonucleoprotein complexes containing human LINE-1 protein and RNA. EMBO J. 15: $630-639$.

Holmes, S.E., Singer, M.F., and Swergold, G.D. 1992. Studies on p40, the leucine zipper motif-containing protein encoded by the first open reading frame of an active human LINE-1 transposable element. $J$. Biol. Chem. 267: 19765-19768.

Houseley, J., LaCava, J., and Tollervey, D. 2006. RNA-quality control by the exosome. Nat. Rev. Mol. Cell Biol. 7: 529-539.

Hulme, A.E., Kulpa, D.A., Garcia-Perez, J.L., and Moran, J.V. 2006. The impact of LINE-1 retrotransposition on the human genome. In Genomic disorders: The genomic basis of disease (eds. J. Lupski and P. Stankiewicz). Humana Press, Totowa, NJ.

Jensen, S. and Heidmann, T. 1991. An indicator gene for detection of germline retrotransposition in transgenic Drosophila demonstrates RNA-mediated transposition of the LINE I element. EMBO J. 10: $1927-1937$.

Jurka, J., Kapitonov, V.V., Pavlicek, A., Klonowski, P., Kohany, O., and Walichiewicz, J. 2005. Repbase Update, a database of eukaryotic repetitive elements. Cytogenet. Genome Res. 110: 462-467.

Kazazian Jr., H.H. and Moran, J.V. 1998. The impact of L1 retrotransposons on the human genome. Nat. Genet. 19: 19-24.

Kent, W.J. 2002. BLAT-The BLAST-like alignment tool. Genome Res. 12: $656-664$.

Kimberland, M.L., Divoky, V., Prchal, J., Schwahn, U., Berger, W., and Kazazian Jr., H.H. 1999. Full-length human L1 insertions retain the capacity for high frequency retrotransposition in cultured cells. Hum. Mol. Genet. 8: 1557-1560.

Kulpa, D.A. and Moran, J.V. 2005. Ribonucleoprotein particle formation is necessary but not sufficient for LINE-1 retrotransposition. Hum. Mol. Genet. 14: 3237-3248.

Kulpa, D.A. and Moran, J.V. 2006. Cis-preferential LINE-1 reverse transcriptase activity in ribonucleoprotein particles. Nat. Struct. Mol. Biol. 13: 655-660. 
Kunkel, G.R. and Pederson, T. 1988. Upstream elements required for efficient transcription of a human U6 RNA gene resemble those of $\mathrm{U} 1$ and $\mathrm{U} 2$ genes even though a different polymerase is used. Genes \& Dev. 2: 196-204.

Lobo, S.M. and Hernandez, N. 1989. A 7 bp mutation converts a human RNA polymerase II snRNA promoter into an RNA polymerase III promoter. Cell 58: 55-67.

Luan, D.D., Korman, M.H., Jakubczak, J.L., and Eickbush, T.H. 1993. Reverse transcription of R2Bm RNA is primed by a nick at the chromosomal target site: A mechanism for non-LTR retrotransposition. Cell 72: 595-605.

Martin, S.L. and Bushman, F.D. 2001. Nucleic acid chaperone activity of the ORF1 protein from the mouse LINE-1 retrotransposon. Mol. Cell. Biol. 21: 467-475.

Martin, S.L., Branciforte, D., Keller, D., and Bain, D.L. 2003. Trimeric structure for an essential protein in L1 retrotransposition. Proc. Natl. Acad. Sci. 100: 13815-13820.

Martin, S.L., Cruceanu, M., Branciforte, D., Wai-Lun Li, P., Kwok, S.C., Hodges, R.S., and Williams, M.C. 2005. LINE-1 retrotransposition requires the nucleic acid chaperone activity of the ORF1 protein. $J$. Mol. Biol. 348: 549-561.

Mathias, S.L., Scott, A.F., Kazazian Jr., H.H., Boeke, J.D., and Gabriel, A 1991. Reverse transcriptase encoded by a human transposable element. Science 254: 1808-1810.

Moran, J.V., Holmes, S.E., Naas, T.P., DeBerardinis, R.J., Boeke, J.D., and Kazazian Jr., H.H. 1996. High frequency retrotransposition in cultured mammalian cells. Cell 87: 917-927.

Morrish, T.A., Gilbert, N., Myers, J.S., Vincent, B.J., Stamato, T.D. Taccioli, G.E., Batzer, M.A., and Moran, J.V. 2002. DNA repair mediated by endonuclease-independent LINE-1 retrotransposition. Nat. Genet. 31: 159-165.

Ohshima, K. and Okada, N. 2005. SINEs and LINEs: Symbionts of eukaryotic genomes with a common tail. Cytogenet. Genome Res. 110: $475-490$.

Ostertag, E.M., Prak, E.T., DeBerardinis, R.J., Moran, J.V., and Kazazian Jr., H.H. 2000. Determination of L1 retrotransposition kinetics in cultured cells. Nucleic Acids Res. 28: 1418-1423.

Ostertag, E.M., Goodier, J.L., Zhang, Y., and Kazazian Jr., H.H. 2003. SVA elements are nonautonomous retrotransposons that cause disease in humans. Am. J. Hum. Genet. 73: 1444-1451.

Perreault, J., Noel, J.F., Briere, F., Cousineau, B., Lucier, J.F., Perreault, J.P., and Boire, G. 2005. Retropseudogenes derived from the human Ro/SS-A autoantigen-associated hY RNAs. Nucleic Acids Res. 33: 2032-2041.

Sassaman, D.M., Dombroski, B.A., Moran, J.V., Kimberland, M.L., Naas, T.P., DeBerardinis, R.J., Gabriel, A., Swergold, G.D., and Kazazian Jr., H.H. 1997. Many human L1 elements are capable of retrotransposition. Nat. Genet. 16: 37-43.

Schmitz, J., Churakov, G., Zischler, H., and Brosius, J. 2004. A novel class of mammalian-specific tail-less retropseudogenes. Genome Res. 14: 1911-1915.

Scott, A.F., Schmeckpeper, B.J., Abdelrazik, M., Comey, C.T., O’Hara, B., Rossiter, J.P., Cooley, T., Heath, P., Smith, K.D., and Margolet, L. 1987. Origin of the human L1 elements: Proposed progenitor genes deduced from a consensus DNA sequence. Genomics 1: 113-125.

Van Arsdell, S.W., Denison, R.A., Berstein, L.B., Weiner, A.M., Manser, T., and Gesteland, R.F. 1981. Direct repeats flank three small nuclear RNA pseudogenes in the human genome. Cell 26: 11-17.

Wallace, M.R., Andersen, L.B., Saulino, A.M., Gregory, P.E., Glover, T.W., and Collins, F.S. 1991. A de novo Alu insertion results in neurofibromatosis type 1 . Nature 353: 864-866.

Wang, H., Xing, J., Grover, D., Hedges, D.J., Han, K., Walker, J.A., and Batzer, M.A. 2005. SVA elements: A hominid-specific retroposon family. J. Mol. Biol. 354: 994-1007.

Weber, M.J. 2006. Mammalian small nucleolar RNAs are mobile genetic elements. PLoS Genet. 2: e205.

Wei, W., Morrish, T.A., Alisch, R.S., and Moran, J.V. 2000. A transient assay reveals that cultured human cells can accommodate multiple LINE-1 retrotransposition events. Anal. Biochem. 284: 435-438.

Wei, W., Gilbert, N., Ooi, S.L., Lawler, J.F., Ostertag, E.M., Kazazian, H.H., Boeke, J.D., and Moran, J.V. 2001. Human L1 retrotransposition: cis preference versus trans complementation. Mol. Cell. Biol. 21: 1429-1439.

Received August 16, 2006; accepted in revised form February 2, 2007. 


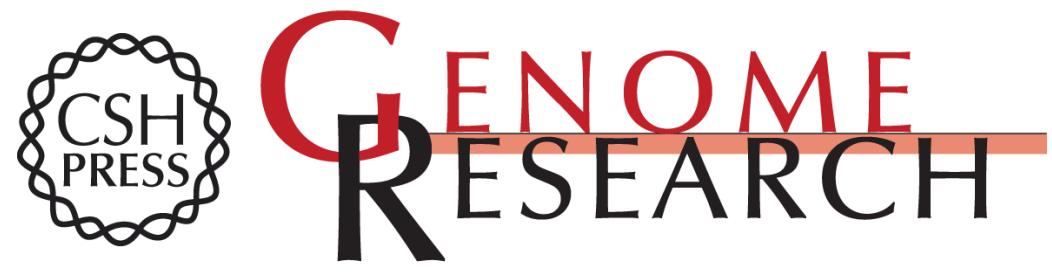

\section{Distinct mechanisms for trans-mediated mobilization of cellular RNAs by the LINE-1 reverse transcriptase}

José L. Garcia-Perez, Aurélien J. Doucet, Alain Bucheton, et al.

Genome Res. 2007 17: 602-611 originally published online April 6, 2007

Access the most recent version at doi:10.1101/gr.5870107

Supplemental http://genome.cshlp.org/content/suppl/2007/04/06/gr.5870107.DC1

Material

References This article cites 57 articles, 22 of which can be accessed free at:

http://genome.cshlp.org/content/17/5/602.full.html\#ref-list-1

\section{License}

Email Alerting Receive free email alerts when new articles cite this article - sign up in the box at the Service top right corner of the article or click here.

\section{Affordable, Accurate Sequencing.}

To subscribe to Genome Research go to:

https://genome.cshlp.org/subscriptions 